\title{
A Learning Assistance System for the Ergonomic Behavioural Prevention in Production
}

\author{
Justus Brosche, Hannes Wackerle, Peter Augat, Hermann Lödding
}

\section{Introduction}

Musculoskeletal disorders (MSDs) are the major cause for incapacity for work in the German production industry (BAuA 2019), and health problems of the upper extremeties and lower back account for around $39 \%$ of occupational diseases in Europe (van Eerd et al. 2016). Thus, ergonomic work processes are especially important in order to protect the workers' health and reduce high follow-up costs for companies and society.

Therefore, on the one hand, it is necessary to make workplaces more ergonomic (so-called organisational prevention). On the other hand, workers need to be trained how to carry out work processes ergonomically and thus optimise their individual behaviour at the workplace (so-called behavioural prevention) (HalbeHaenschke 2017). While digital ergonomics analyses for organisational prevention increase in popularity, modern technologies are mainly used to control different health risk factors in behavioural prevention, such as body weight, nutrition and physical activity (Pfaff/Zeike 2017).

A comprehensive ergonomics analysis would account for the workers' individual physical ability, ergonomic work processes as well as the individual strain on the worker caused by the work processes. However, with the methods available today, it would be too time-consuming and often too slow to allow short-cycle learning.

The goal of the learning assistance system (LAS) described below is to enable such an analysis with the help of a motion capture system. For this purpose, an employee trained in the method carries out a capability and workplace analysis with the production worker.

The paper is structured as follows: chapter 2 describes the theoretical background and chapter 3 shows the LAS' structure. Subsequently, the capability analysis (chapter 4) and workplace analysis (chapter 5) are presented. The paper closes with a summary and outlook (chapter 6). 


\section{Theoretical Background}

\subsection{Behavioural and organisational prevention}

Behavioural prevention aims to minimise behaviour that poses a risk to health and to promote health literacy as well as healthy and safe behaviour. Corporate health management usually focusses on information events, presentations, workshops and seminars for behavioural prevention (Halbe-Haenschke 2017) even though this traditional form of health education and instruction shows little effect (Richter/Rosenbrock 2014). This is due to the fact that little use is made of behavioural preventive measures and that they often do not reach the population groups with the greatest need (Jordan 2020).

Organisational prevention addresses the creation of a health-promoting environment. In ergonomics, the focus lies on ergonomic workplace design (Halbe-Hanschke 2017). In order to improve a workplace according to ergonomic aspects, a risk assessment needs to be carried out first. Occupational physical stress is assessed using screening methods, such as the Key Indicator Methods, the European Assembly Worksheet or the Rapid Upper Limb Assessment (RULA). The results can then be used to derive measures for an improved work design (BAuA 2019).

Because RULA assesses individual body postures and focusses on the upper extremities, it is particularly suitable to assess the individual behaviour in production processes. Based on selected joint angles and loads acting on the body, it determines a risk value that describes the risk of suffering an MSD. By relating it to specific body postures, RULA makes it easier to derive effective countermeasures (McAtamney/Nigel Corlett 1993).

\subsection{Virtual Ergonomics}

In the course of technological progress, virtual ergonomics is becoming increasingly important. With the help of digital human models (DHMs), computer-aided methods and tools can be used for the ergonomic design of products, work systems and processes (Mühlstedt 2016a). DHMs represent the geometry and other human properties and allow a digital manipulation of body postures using forward or inverse kinematics. In addition, it is possible to record human movements with motion capture systems and impose them on a DHM (Mühlstedt 2016b). The technologies mentioned above can be used to digitise the screening methods for organisational prevention described in 2.1. However, there are only a few approaches that use these methods for behavioural prevention, for example by evaluating the body posture in real time with a 3D camera and then providing feedback to the worker (e. g. Martin et al. 2012). 


\section{Production}

\subsection{Capability Analyses}

In the health sector and ergonomics, capability analyses are described as functional capacity evaluations. Two comprehensive methods have been established to determine the physical capabilities of an employee: the Functional Capacity Evaluation according to Isernhagen and the ERGOS work simulator (Oliveri 2006). Both methods use standardised tests to assess the subject's work-related physical capacity. Since they are very costly ( $\sim 5$ hours excluding the time for evaluations and reports), assessing the capabilities of an entire workforce is expensive and timeconsuming. These methods are therefore mostly applied in occupational therapy contexts, for example, to secure a damage-free return to work after an illness or to quantify the course of rehabilitation (Kaiser et al. 2000; Oliveri 2006).

\subsection{Mobility Analyses}

Complex three-dimensional movements or movements in two dimensions can be used to assess mobility. When focusing on complex movements, it is often not obvious which joints and movements are deficient. Movement goals can be reached by means of compensatory movements, although a certain joint function is not given. If only the movement in $3 \mathrm{D}$ is analysed, joint angle maxima and mobility deficits around a movement axis may not be recognised. A reliable statement on the mobility of a joint is only possible by testing the range of motion (ROM) in $2 \mathrm{D}$, as no compensatory movements influence the quality of movement. The problems of correctly recording joint mobility can be illustrated by the example of the shoulder:

The structure of the shoulder joint is a compromise between mobility and stability. A completely isolated movement in one plane is not possible since it is always a combination of multiple movements. During flexion and extension of the joint, there is always also a rotation of the humeral head and translations in the glenohumeral joint (Novotny et al. 1998; Stokdijk et al. 2003).

For the representation of joint mobility, the neutral zero method has become established. It is a standardised method to measure joint mobility and is based on movements in each plane of motion around the corresponding axis. For each degree of freedom, two different motions are possible, such as the flexion and extension or abduction and adduction of a joint (Schünke et al. 2018).

\subsection{Correlation between grip strength and global muscle strength}

Studies (Porto et al. 2019) show a significant positive correlation between handgrip strength and global muscle strength. Global muscle strength has various yet similar definitions: Porto et al. (2019) define it as the summation of the peak muscle torques of the trunk and dominant muscle groups of the lower extremities (see Figure 1). Tietjen-Smith et al. (2006) measure the global muscle strength by summing 
strength measures like shoulder press, bench press, back pull-down and knee extension and flexion. Wind et al. (2010) define it as the sum of shoulder abductors, hip flexors and ankle dorsiflexors. The different studies show significant positive correlations between grip strength and multiple muscle groups for healthy adults of both genders. Grip strength correlates significantly with the knee extensors and flexors and back strength and also with the maximum muscle torques of the trunk, hips, knees and ankles. Furthermore, there are gender-dependent differences. Men have higher handgrip strength values than women and the maximum handgrip strength decreases with age for both genders, after the maximum was reached between 30 and 40 years (Steiber 2016).

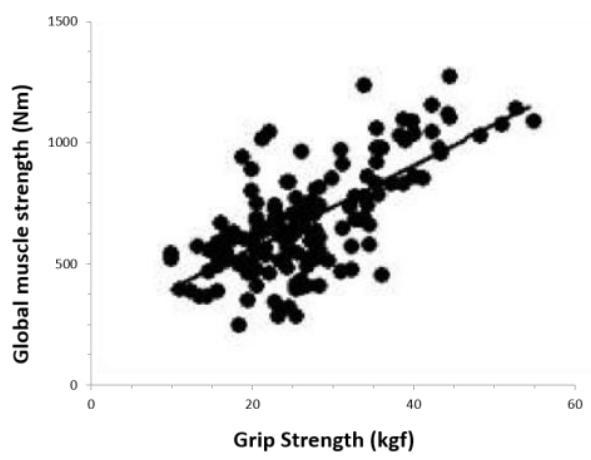

Figure 1: Correlation between global muscle strength and grip strength. Adapted from Porto et al. (2019).

\section{Concept for the LAS}

This chapter describes the structure of the proposed LAS, the derived requirements and the utilised hardware and software.

\subsection{Structure}

Figure 2 shows the schematic structure of the LAS. In the capability analysis, worker mobility and strength are measured in order to create a physical capability profile. This physical capability profile shows the worker's ability to move and his or her load-bearing capacity. In the course of the workplace analysis, the work processes and external loads acting on the worker are recorded in order to determine the workplace-induced stress. By comparing the worker's physical capabilities with the workplace-induced stress, the workplace-induced strain on the individual worker can be determined. This approach allows to derive different measures:

- Individual measures to generally increase the worker's strength and mobility can be derived from the employee's capabilities. 
Workplace-specific measures for all employees can be derived from the workplace-induced stress.

Workplace-specific measures for individual worker can be derived from the strain.

\begin{tabular}{|c|c|c|c|}
\hline Acquisition & mobility \& strength & & $\begin{array}{c}\text { work processes \& } \\
\text { external loads }\end{array}$ \\
\hline Analysis & capability analysis & & workplace analysis \\
\hline Result & worker capability & strain & $\begin{array}{c}\text { workplace-induced } \\
\text { stress }\end{array}$ \\
\hline Measure & individual & $\begin{array}{c}\text { individual \& } \\
\text { workplace-specific }\end{array}$ & workplace-specific \\
\hline
\end{tabular}

Figure 2: Schematic structure of the LAS

The structure and the field of application result in the following requirements for the capability and workplace analysis for an application in the LAS:

In order to meet the specific conditions of a company and to create a physical capability profile for an entire workforce or multiple chosen workers, the capability analysis has to be carried out in a significantly lower timeframe than the methods presented earlier. However, the capability profile needs to give a comprehensive picture of the individual worker's physical capabilities. The research goal was to find a suitable compromise between analysis effort and quality and to develop a method to measure the capabilities.

In order to prevent MSDs effectively, the workplace ergonomics needs to be assessed at an early stage. As described in section 2.1, existing ergonomics analyses aim at organisational prevention. The focus of the LAS is to improve the worker's behaviour at the workplace. Thus, it is necessary to assess individual postures and not the workplace as a whole. Furthermore, the analysis results have to be processed and visualised comprehensively to promote short learning cycles and quickly verify the effect of derived measures.

The use of the LAS requires two people: a staff member trained in the method and the production worker to be examined. The identification of critical work processes and the derivation of suitable measures require an advanced understanding of ergonomics, which is why it is generally not advisable for the production worker to use the system alone.

\subsection{Utilised Hardware and Software}

The kinematic data is captured with the XSens MVN Awinda motion capture system (Xsens MVN Awinda, Xsens Technology BV, Enschede, Netherlands). In contrast to camera-based systems, the inertial sensor system allows motion analysis 
at the workplace since the recording quality is not impaired by the occlusion of body parts by workpieces or machines. The kinematic information of the recorded human motion sequence (e.g. the position of 23 body segments) is extracted and converted using the corresponding software.

The software for the LAS was developed using the game engine Unity and allows a quick evaluation of the capability and workplace analysis. The external loads are tracked with a web application that records the weight class and the time stamp of grabbing and releasing workpieces or tools.

\section{Capability Analysis}

As described in section 3.1, a capability analysis needs to be performed in order to derive individual measures that improve the worker's capabilities if deficits are noted and adjust the work task to his or her needs. This chapter describes the capability analysis that is used to determine a worker's ability to move and his or her load-bearing capacity. The capability analysis comprises 14 standardised movement exercises (see Table 1) to assess joint mobility, and a grip strength measurement to estimate the global muscle status. The analysis takes approximately 30 minutes in total, which already includes taking body measurements and putting on the motion capture system. In order to minimise the time requirement, the use of a 3D camera for the mobility analysis was examined (Brosche et al. 2020) but the tracking algorithms could not achieve satisfactory results for all movement exercises. If technological progress allows the use of a 3D camera to record the movement exercises, the analysis duration can be reduced to 10 to 15 minutes.

4.1. Standardised movement exercises and application of the neutral zero method

For the mobility analysis, standardised movement exercises (see Table 1) are performed to identify deficits of single joints. The standardised exercises help to objectify existing restrictions by comparing the results of the neutral zero method with a standard ROM assessment. The individual mobility of the assessed joints can thus be compared to standard values from literature. Consequently, it is possible to provide individual feedback to the worker and assess his or her ability to move.

\begin{tabular}{|l|c|c|}
\hline Joint & \multicolumn{3}{|c|}{ Movement } \\
\hline Cervical spine & Flexion/ Extension & Lateral flexion \\
& &
\end{tabular}




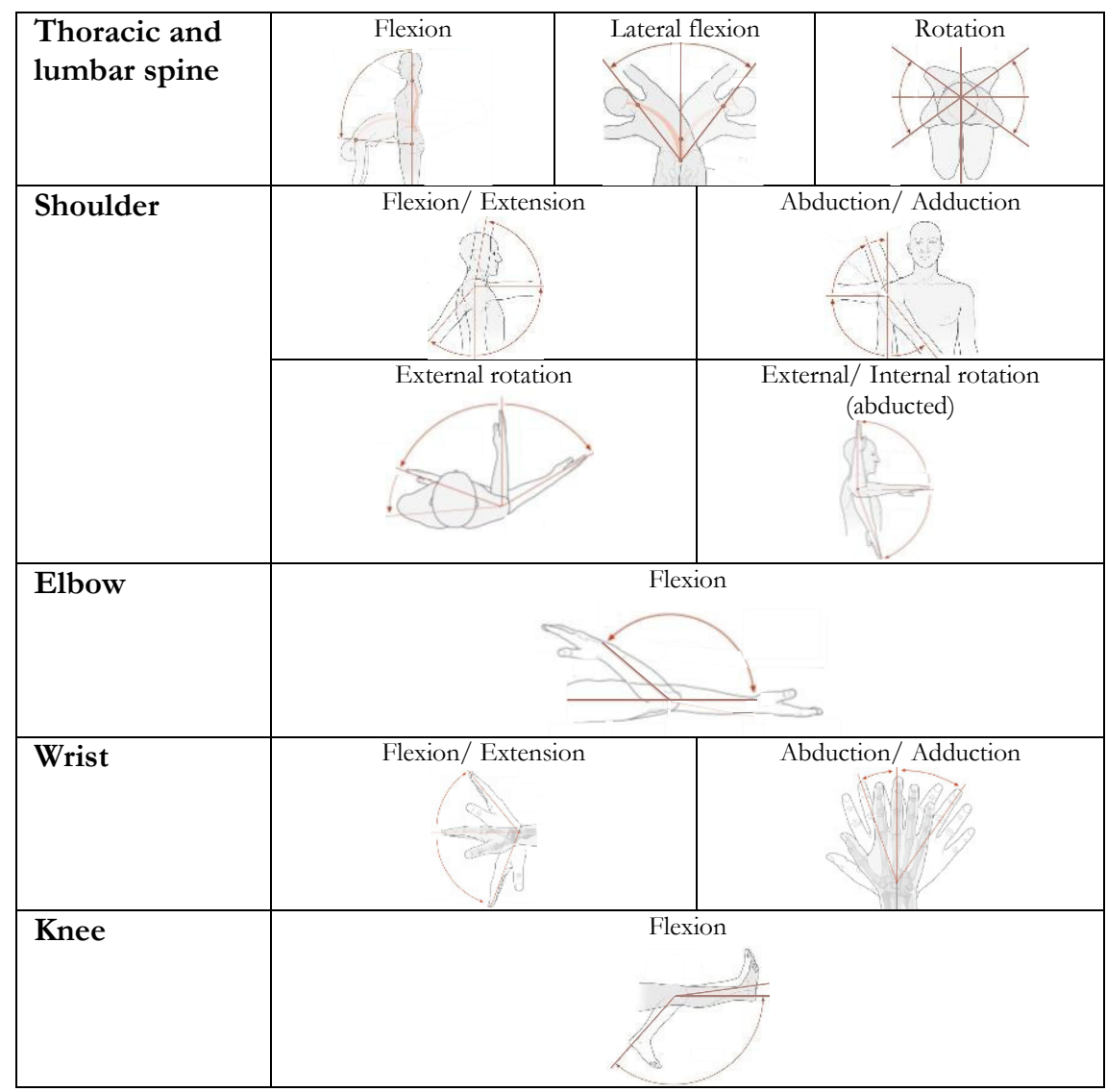

Table 1: Standardised movement exercises. Adapted from Schünke et al. (2018).

\subsection{Grip strength indicating the load-bearing capacity}

There is a significant positive correlation between handgrip strength and total muscle strength (see section 2.5). Therefore, the evaluation of grip strength can be used for an assessment of physical fitness or health status. The grip force is examined by means of a hand force dynamometer, an inexpensive standard measuring device. In comparison to the ERGOS work simulator, where the strength ability is tested on a larger scale, here the strength measurement is limited to the hand strength alone.

By using the measurement of grip strength as part of the capability analysis, statements can be made about the muscle status and health status of individual workers. 
These individual statements can then be used to adapt global load limits to individual workers (see section 5.1).

\subsection{Application of the capability analysis in the LAS}

With the help of the mobility and hand force measurements from the capability analysis, a capability profile of the employee is created. In the LAS, the capability profile is initially viewed in isolation. With the help of a 3D representation of the generated DHM, possible limitations in mobility can be visualised in an easily understandable way and conspicuous joints can be quickly identified (see Figure 3). The joints are represented by spheres which are coloured based on the results of the mobility analysis. The joints with corresponding angles below the respective normal range are coloured yellow and the measured values are shown in relation to the lower limit of the normal range. Excessive joint mobility is not indicated due to the unrestricted movement execution. When discussing the results, particular attention needs to be paid to high deviations from the normal values and intraindividual differences.

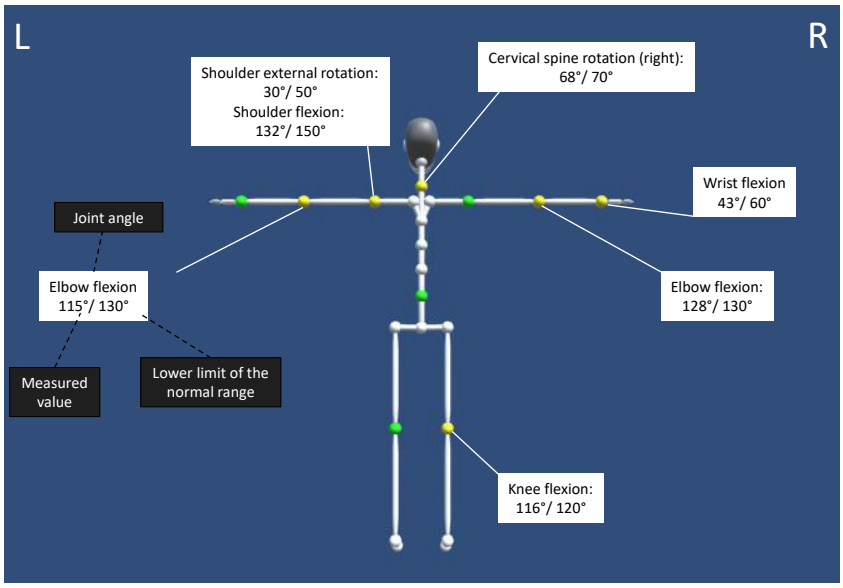

Figure 3: A traffic light system to visualise joint mobility

In the example in Figure 3, several measured values are below the standard ROM. Slightly falling below the lower limit of the normal range does not require any measures to be taken. This is the case for the cervical spine rotation, the right elbow flexion and right knee flexion from the example. In the case of greater deviations from the normal range, a follow-up examination by the company physician should follow. In the example, this would be the case for the external rotation and flexion of the left shoulder and for the left elbow flexion.

In addition, the grip strength measurement is discussed with the employee. Possible restrictions are only registered when a measured value for the right or left hand 


\section{Production}

is below the age-specific normal value minus its standard deviation and can thus be classified as significantly low. If there are any abnormalities, a visit to the company doctor is recommended in order to carry out more detailed examinations. If necessary, targeted strength training could be initiated to improve overall muscle strength and the overall health status. By repeating the capability analysis regularly (e. g. every year), the workers' physical capabilities can be monitored and the effect of strength or mobility training can be checked.

The full capability analysis has so far been carried out with a sample of 7 people aged 33 to 56 in industry. In this small sample, several limitations in mobility were found and two significant deviations of hand strength from the normal value could be identified.

\section{Workplace Analysis}

After assessing the worker's individual capabilities, the workplace analysis is used to determine the stress of the workplace and the resulting physical strain on the worker. In order to meet the requirements mentioned in section 3.1, the workplace analysis consists of two different modules: The physical stress is determined with the help of the RULA and the calculation of joint torques. The strain results from the comparison of the worker's mobility with the joint angles occurring in the work process and by individualising the global joint torque limits in relation to the grip strength.

\subsection{Physical stress}

RULA is used as a tool for workplace analysis, as it enables an assessment of individual postures, focuses on the upper body and includes external loads in the ergonomics assessment. For the software implementation, the methodology described by McAtamney/Nigel Corlett (1993) was implemented with three restrictions. In order to keep the collection of metadata as simple as possible, arm support/ leaning and extra points due to static posture or repetition are not recorded. In addition, all loads are assumed to be intermittent. Joint angles that do not have a specified range are recorded if the measured values deviate by more than ten degrees from zero. The shoulder is considered abducted if the angle exceeds 60 degrees.

To calculate the joint torques, the external loads moved by the workers and the kinematic data of all body segments acting on the joint are required as input data. The masses of the body segments are standardised on the basis of the individual body weight according to Winter (1990). Each body segment is assumed to be cylindrical and the centre of mass is assumed to be central.

With the help of the Varignon's theorem, the joint torque can be calculated from the input data and classified using limit values for the assessment. The quasi-static 
biomechanical analysis of the individual joint torques allows a risk estimation of suffering MSDs. The German Social Accident Insurance (DGUV 2015) defines the torque limit values for the lower lumbar spine assessment (L5/S1-torque) and employs a traffic light system as follows:

$0-40 \mathrm{Nm}$; green area; everything is ok; no need for action

- 40 - $80 \mathrm{Nm}$; yellow area; slightly increased joint torques; measures for people with restriction might be necessary

$80-135 \mathrm{Nm}$; orange area; increased joint torques; need for action

$>135 \mathrm{Nm}$; red area; strongly increased joint torques; peak load must be reduced by counter measures

The following assumptions were made for the calculation of joint torques:

The process is regarded as quasi-static.

- Weights are recorded from $3 \mathrm{~kg}$ and divided into weight classes (3 - $5 \mathrm{~kg}$, $5-10 \mathrm{~kg}, 10-15 \mathrm{~kg}, \ldots,>40 \mathrm{~kg})$. The torques are calculated rounding to the upper limit.

The load acts in the centre of the hand.

When handling loads with both hands, $100 \%$ of the load acts on each shoulder, as it is not possible to assess the force distribution from the outside. In the torque calculation for the lower lumbar spine, the load is distributed $50 \%$ to each hand.

- Body segments are assumed to be cylindrical with a central centre of gravity. Their weights are derived from standard percentages (Winter 1990).

\subsection{Physical strain}

After the physical stress assessment to identify the load on all workers, a physical strain assessment is performed to evaluate the effects on the individual worker and his or her capabilities. As described in section 4.1, the ability to move is determined using standard exercises with two-dimensional movement execution. In order to establish comparability between the complex movements occurring in the process and joint mobility, all movements are transformed into the same planes from the movement exercise. For example, a complex movement of the shoulder joint is divided into shoulder flexion/extension and shoulder abduction/adduction. The software calculates the percentage utilisation of joint mobility as a characteristic value. 


\section{Production}

In order to determine the strain on the load-bearing capacity, the limit values for the joint torque evaluation are reduced if the worker's hand strength is significantly low. The grip strength of each hand influences the limit value of the respective shoulder torque and the minimum grip strength affects the limit value for the lower lumbar spine torque. The limit values are not increased if a high grip strength is measured.

\subsection{Application of the workplace analysis in the LAS}

With the help of the above-mentioned analyses, the stress and strain at the workplace can be determined and discussed with the employee. A DHM is used for visualisation. Here, twists in the spine and joint movements that are otherwise hidden by clothing can be easily recognised. In addition, the software allows camera movement in $3 \mathrm{D}$ space, which means that there is no occlusion and body postures can be viewed from any angle.

Figure 4 shows an exemplary posture to which the following section refers. In this example, the worker handles a weight of $15 \mathrm{~kg}$ with both hands. In addition to the shown DHM and the traffic light system for the torque assessment, the strain on the ability to move and RULA-scores are visualised. In order to identify ergonomically critical points in the work process, work steps with high RULA values, high joint torques and/or high percentage utilisation of one or more joint angles are automatically highlighted. In the following, only measures for ergonomic behavioural prevention are discussed. In industrial use, it should be checked for each posture whether an organisational preventive measure in the form of a workplace improvement would be more useful.

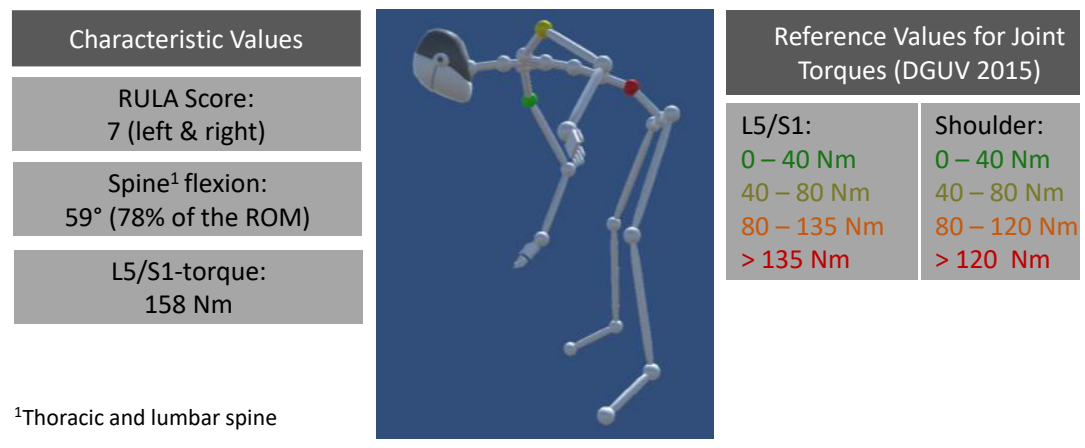

Figure 4: Exemplary posture while handling $15 \mathrm{~kg}$ 


\section{Workplace-induced stress}

When considering workplace-induced stress, the RULA values and the driving factors are considered first. In the example, the RULA results in the maximum risk value for the left and right halves of the body. The driving factors are the body posture, i.e. the posture of the cervical spine and the upper body, and the high external weight. From this observation, it can already be deduced as a first behavioural preventive measure that the worker should lift loads that are placed close to the ground with his or her legs instead of the back.

Subsequently, the joint torques are considered and evaluated with the global limit values from section 5.1. The load on the lower lumbar spine supports the results of the RULA and underlines the importance of posture correction. In the red zone, damage to the musculoskeletal system can already occur due to short-term peak loads, for example in the form of a herniated disc. The aim of this observation is to increase the employee awareness to the fact that even light weights in combination with non-ergonomic postures lead to a high load on the body. This should create awareness to pay attention to one's movement even with supposedly light weights and to use provided aids, such as a crane for load handling. The shoulder torques are within an acceptable range, with a slightly increased joint torque for the left shoulder.

\section{Workplace-induced strain}

In contrast to stress, which describes the external effects on people, by loads to be moved and the design and equipment of the workplace, strain is described as the reaction of people to such stresses. The degree of stress is strongly dependent on individual prerequisites, which can be determined by means of the capability analysis described above.

First, the stress on the joint mobility is considered. In the present example, none of the joints considered are close to their maximum ROM. However, it should be noted that the flexion of the thoracic and lumbar spine is already at $78 \%$ capacity. Since the work step is not a long-lasting movement, no behavioural preventive measure can be derived from this. In addition to adapting the movement sequence, measures to increase mobility, e.g. in the form of sports or stretching exercises, may be recommended if the strain was greater.

In the last step, the joint torques are considered again, with the difference that the limit values for the evaluation of the joint torques are now reduced depending on the hand strength measurement. If the worker had a grip strength measurement significantly below average for the left hand, lowering the limit values of the left shoulder torque leads to a new evaluation and a classification of the joint torque in the orange area (see section 5.1). In this case, exercises to strengthen the upper body would be recommended. The double consideration of joint torques aims at a higher awareness on the part of the worker that his or her individual capabilities 
have a significant influence on the effect of workplace stress and that low overall muscle strength requires an expanded health awareness at the workplace.

Following the analysis of each work step, the workplace analysis can be repeated to demonstrate the effect of immediate measures such as improved movement execution. Individual movements should be performed again directly after the measures have been derived and before the next critical work step in order to achieve a high learning effect. The learning success can be evaluated by repeating the assessment regularly (e.g. every year). If the same work process is analysed, the characteristic values of the initial recording can be compared with the characteristic values of the repeated recording to quantify the effect of the LAS on the workers' behaviour.

The workplace analysis was carried out with the same test persons as in section 4.3 at multiple assembly and logistics workplaces. The stress and strain on the employees was determined, but a review of the measures derived in the learning assistance system has not yet been carried out.

\section{Summary and Outlook}

This paper shows how a low-effort capability analysis can be carried out with 14 movement exercises and a grip strength measurement to create an ergonomic capability profile for production workers. With a workplace analysis, which includes the RULA and a consideration of joint torques, the workplace-induced stress is determined. A combination of the two analyses leads to workplaceinduced strain on the worker. Through the low-effort recording with a motion capture system and through an automated evaluation, the results can be used in a LAS for ergonomic behavioural prevention.

The direct individualised ergonomic evaluation is visualised with the help of DHMs. This makes it possible to identify non-ergonomic movements at the workplace, to improve them and to review the measure. In addition, measures can be recommended to the employee to increase his or her mobility and strength capabilities in order to avoid the overload of individual capabilities and to enable safe work. The learning success can be monitored by repeating the assessment in certain intervals.

The individual elements of the LAS have already been tested in an industrial environment, but the concept has yet to be implemented as a whole over a longer period of time. Also, it needs to be checked whether the hand strength is sufficient as an indicator of the load-bearing capacity. Although the correlation of hand strength with total muscle strength indicates the legitimacy of the assumption, a 
clinical study should be carried out on the correlation between hand strength and the limit values of the joint torques.

\section{Acknowledgements}

The research project ErgoTrack (20465 N) was funded by the German Federal Ministry of Economics and Energy due to a decision of the German Bundestag. The authors would like to thank the German Federation of Industrial Research Associations $(\mathrm{AiF})$ and the companies involved in the research project.

\section{References}

BAuA (2019). MEGAPHYS - Mehrstufige Gefährdungsanalyse physischer Belastungen am Arbeitsplatz. Dortmund: Bundesanstalt für Arbeitsschutz und Arbeitsmedizin. https://doi.org/10.21934/baua:bericht20190821

Brosche, J., Wackerle, H., Lödding, H. (2020). Creating a Worker-Individual Physical Ability Profile Using a Low-Cost Depth Camera. In Nyhuis, P., Herberger, D., Hübner, M. (Hrsg.), Proceedings of the 1st Conference on Production Systems and Logistics (CPSL 2020) (S. 269-276).

DGUV (Hrsg.) (2015). Bewertung physischer Belastungen gemäß DGUV-Information 208-033 (bisher: BGI/GUV-I 7011) (Anhang 3). Berlin.

Halbe-Haenschke, B. (2017). Informieren: Was ist BGM. In Halbe-Haenschke, B., Reck-Hog, U. (Hrsg.), Die Erfolgsstrategie für Ihr BGM (S. 17-31). Wiesbaden: Springer Fachmedien Wiesbaden. https://doi.org/10.1007/978-3-658-15491-2_3.

Pfaff, H., Zeike, S. (2017). Digitalisierung von Arbeit und Gesundheit: ein Überblick. In Knieps, F., Pfaff, H. (Hrsg.), BKK Gesundheitsreport: Vol. 41.2017. Digitale Arbeit - digitale Gesundheit: Zahlen, Daten, Fakten: mit Gastbeiträgen aus Wissenschaft, Politik und Praxis (S. 25-30). Berlin: Medizinisch Wissenschaftliche Verlagsgesellschaft.

Jordan, S. (2020). Inanspruchnahme präventiver Maßnahmen. Eine Analyse von Einflussfaktoren auf die Nutzung von Verhaltensprävention bei Erwachsenen. Abgerufen von https://pub.uni-bielefeld.de/record/2941520. https://doi.org/10.4119/unibi/ 2941520

Kaiser, H., Kersting, M., Schian, H.-M., Jacobs, A., Kasprowski, D. (2000). Der Stellenwert des EFLVerfahrens nach Susan Isernhagen in der medizinischen und beruflichen Rehabilitation. Rehabilitation, 39, 297-306. https://doi.org/10.1055/s-2000-7861

Martin, C. C., Burkert, D. C., Choi, K. R., Wieczorek, N. B., McGregor, P. M., Herrmann, R. A., Beling, P. A. (2012). A real-time ergonomic monitoring system using the Microsoft Kinect. In 2012 IEEE Systems and Information Engineering Design Symposium. 
McAtamney, L., Nigel Corlett, E. (1993). Rula: A survey method for the investigation of work-related upper limb disorders. Applied Ergonomics, 24(2), 91-99. https://doi.org/10.1016/00036870(93)90080-s

Mühlstedt, J. (2016a). Grundlagen virtueller Ergonomie. In Bullinger-Hoffmann, A. C., Mühlstedt, J. (Hrsg.), Homo Sapiens Digitalis - Virtuelle Ergonomie und digitale Menschmodelle (S. 7-39). Berlin, Heidelberg: Springer Berlin Heidelberg. https://doi.org/10.1007/978-3-662-50459-8_2

Mühlstedt, J. (2016b). Digitale Menschmodelle. In Bullinger-Hoffmann, A. C., Mühlstedt, J. (Hrsg.), Homo Sapiens Digitalis - Virtuelle Ergonomie und digitale Menschmodelle (S. 73-182). Berlin, Heidelberg: Springer Berlin Heidelberg. https://doi.org/10.1007/978-3-662-50459-8_4

Novotny, J. E., Nichols, C. E., Beynnon, B. D. (1998). Normal kinematics of the unconstrained glenohumeral joint under coupled moment loads. Journal of Shoulder and Elbow Surgery, 7(6), 629-639. https://doi.org/10.1016/s1058-2746(98)90013-1

Oliveri, M. (2006). Functional Capacity Evaluation (FCE). In Gobelet, C., Franchignoni, F. (Hrsg.), Vocational Rehabilitation (S. 69-93). Paris: Springer Paris. https://doi.org/10.1007/2-287-297456_6

Porto, J. M., Nakaishi, A. P. M., Cangussu-Oliveira, L. M., Freire Júnior, R. C., Spilla, S. B., Abreu, D. C. C. de (2019). Relationship between grip strength and global muscle strength in community-dwelling older people. Archives of Gerontology and Geriatrics, 82, 273-278. https://doi.org/10.1016/j.archger.2019.03.005

Richter, M., Rosenbrock, R. (2014). Verhaltensprävention. In Egger, M., Razum, O. (Hrsg.), De Gruyter Studium. Public Health: Sozial- und Präventivmedizin kompakt (2nd ed., S. 132-134). Berlin: De Gruyter. https://doi.org/10.1515/9783110466867-008

Schünke, M., Schulte, E., Schumacher, U., Voll, M., Wesker, K. H. (2018). PROMETHEUS Allgemeine Anatomie und Bewegungssystem (5., vollständig überarbeitete Auflage). PROMETHEUS LernAtlas der Anatomie. Stuttgart, New York: Georg Thieme Verlag. Abgerufen von https://www.thieme-connect.de/products /ebooks/book/10.1055/b-006-149643 https://doi.org/10.1055/b-006-149643

Steiber, N. (2016). Strong or Weak Handgrip? Normative Reference Values for the German Population across the Life Course Stratified by Sex, Age, and Body Height. PLOS ONE, 11(10), e0163917. https://doi.org/10.1371/journal.pone.0163917

Stokdijk, M., Eilers, P. H. C., Nagels, J., Rozing, P. M. (2003). External rotation in the glenohumeral joint during elevation of the arm. Clinical Biomechanics (Bristol, Avon), 18(4), 296-302. https://doi.org/10.1016/s0268-0033(03)00017-2

Tietjen-Smith, T., Smith, S. W., Martin, M., Henry, R., Weeks, S., Bryant, A. (2006). Grip Strength in Relation to Overall Strength and Functional Capacity in Very Old and Oldest Old Females. Physical \& Occupational Therapy in Geriatrics, 24(4), 63-78. https://doi.org/10.1080/J148v24n04_05

Van Eerd, D., Munhall, C., Irvin, E., Rempel, D., Brewer, S., van der Beek, A. J., . . Amick, B. (2016). Effectiveness of workplace interventions in the prevention of upper extremity musculoskeletal disorders and symptoms: An update of the evidence. Occupational and Environmental Medicine, 73(1), 62-70. https://doi.org/10.1136/oemed-2015-102992 
Wind, A. E., Takken, T., Helders, P. J. M., Engelbert, R. H. H. (2010). Is grip strength a predictor for total muscle strength in healthy children, adolescents, and young adults? European Journal of Pediatrics, 169(3), 281-287. https://doi.org/10.1007/s00431-009-1010-4

Winter, D. A. (1990). Biomechanics and motor control of human movement (Second edition). A Wiley-Interscience publication. New York, Chichester, Brisbane: Wiley. Abgerufen von http://www.loc.gov/catdir/description/wiley0310/89070467.html 